\title{
Plausibility of Incoherent Detection for Radiometric Monitoring of Insulation Integrity in HV Substations
}

\author{
J. M. R. de Souza Neto (MIEEE), E. C. T. de Macedo, J. S. \\ da Rocha Neto (SMIEEE), \\ M. F. Queiroz Vieira \\ Departamento de Engenharia Elétrica \\ Universidade Federal de Campina Grande \\ Campina Grande - PB, Brazil \\ \{jose.neto,euler.macedo\}@ee.ufcg.edu.br, \\ zesergio@dee.ufcg.edu.br
}

\author{
I. A. Glover (MIEEE) \\ Department of Engineering \& Technology, \\ University of Huddersfield, Huddersfield, UK \\ i.a.glover@hud.ac.uk \\ M. D. Judd (SMIEEE), R. Atkinson (SMIEEE), J. Soraghan \\ Department of Electronic \& Electrical Engineering \\ University of Strathclyde, Glasgow, UK \\ m.judd@strath.ac.uk
}

\begin{abstract}
The work reported here is part of a $\mathrm{PhD}$ research relates to the development of a new type of radiometric system applied to detection of partial discharges signals. A simulation model for the radiometric transmission of a partial discharge pulse is presented. The model is based on a replay model, which acts as a source, which is based on real data to simulate the pulses. The structure considers losses due to the free space transmission path and interferences caused by carriers from broadcast transmissions. A receiver, focus of this research, is based on a radiometer structure, which includes an amplifier, a filter and an envelope detector to minimize the necessity of a high sample rate ADC.
\end{abstract}

Keywords-Partial Discharge; simulation; radiometer; model; interference.

\section{INTRODUCTION}

Many PD sensors for monitoring the insulation integrity of high voltage (HV) electrical equipment in substations are now available commercially. Most, however, require physical contact with the HV equipment they are monitoring. Sensors such as high frequency current transformers (HFCTs) and transient earth voltage (TEV) detectors rely on near-field inductive, or surface-wave, coupling and therefore require at least one sensor for each item of plant to be monitored.

Recently, a free-standing technology has been developed which allows PD to be detected and located at a distance [1]. This not only removes the need to take HV plant out of service for installation of a monitoring system but can provide area coverage over thousands of square metres from a single sensor. The location method used in this technology is based on time-difference-of-arrival measurements of the partial discharge (PD) signal at three or more spatially separated antennas and signal processing (principally cross-correlation) of the received signals. An alternative approach that could use simpler sensors has been proposed in $[2,3,4]$.

\section{VISION}

The new system being proposed will use amplitude-only measurements of PD signals over an array of antennas leading to a cheaper and more scalable sensor network. The closer a sensor is to a source of PD the larger the PD signal it will be expected to receive. Such a sensor network clearly, therefore, has the potential to both monitor temporal changes in PD (thereby giving warning of possible equipment failure) and locate sources of PD (thereby identifying which item of equipment is at risk of failure). The location of PD sources could (at its most basic) be those items of equipment closest to the sensor node receiving the largest PD signal. More sophisticated location algorithms could use fingerprinting methods to compare the spatial distribution of PD signal amplitudes with that recorded when a calibrated PD-like signal generator is moved systematically through the area that the sensor network is designed to monitor. (Alternatively, permanently installed, low duty-cycle, PD emulators could be distributed through the substation for this calibration function.)

\section{The Challenge}

To realize such a system at least one sensor, and preferable three or more sensors, must be close enough to each potential source of PD to be able to reliably detect significant PD signals. Significant in this case implies signals that are large enough to suggest insulation degradation that might lead to catastrophic failure in less than (say) 12 months. If the spatial distribution of sensors is sufficiently dense then this condition can always be met. The cost of the proposed sensor network, however, should be approximately proportional to the number of nodes. Arbitrarily dense networks are therefore not practical. If, for example, an economic network for a large substation is limited to a few tens of sensors then the range of a sensor must be of the order of $10 \mathrm{~m}$ and preferably (if a single PD source is to be detected by multiple sensors) more than this. Since PD signals are impulsive (pulse durations measured in ns) their bandwidth is large (hundreds of $\mathrm{MHz}$ ). The bandwidth of the sensor receivers will need to be correspondingly large and receiver noise will therefore be high. If the signals are detected incoherently (rather than using correlation between signals in spatially separated receiver), then the signal-to-noise ratio problem, and thus the 
sensitivity/range problem, is clear. Time resolution can mitigate this problem in the following manner. If the individual impulses are detected then the signal-to-noise ratio will be high for the short duration of the impulse (at other times the SNR is zero, i.e. no signal.) This solution is superficially attractive until the challenge of providing an adequately fast ADC is considered. Whilst it is technologically possible to sample the received PD signal sufficiently fast to capture the short duration impulses (during which time SNR is high) the hardware required will become more complex and costly. Worse, the power required to run the fast ADC would be onerous. Furthermore, either additional signal processing at the node will be required or large amounts of raw data will need to be transmitted back to a data collection point for processing. Neither of these options is attractive since both will increase power requirement still further. Nodes will need to operate for long periods (at least 12 months) between maintenance events and battery and energy harvesting technologies (for example) will be able to support only the lowest energy solutions.
A possible compromise between a fast (100s $\mathrm{MHz}-\mathrm{GHz})$ ADC able to detect raw PD impulses (with high SNR) and a slow ADC (sub-Hz) sampling simple power measurement (with low SNR and integration time measured in seconds) might be to use an envelope detector to ensure that impulses decay with a time constant perhaps an order of magnitude less than the expected mean interval between PD pulses [5] (say $10 \mathrm{~s}-100 \mathrm{~s}$ of $\mu \mathrm{s})$. This allows a significantly slower $(10 \mathrm{~s}-$ $100 \mathrm{~s}$ of $\mathrm{kHz}$ ) ADC to be used, which is able to capture the decaying signal at the envelope detector output with sufficient fidelity. The probability of detecting the signal near its peak (and thus securing a high SNR measurement) increases with sampling frequency, as does the cost (in \$) of the ADC and the cost (in watts) of the required power. The envelope detector, therefore, may provide an effective mean to reduce the sampling rate and thus realise a lower-cost, lower-power system.

In order to investigate the plausibility of these arguments a first-order simulation of a PD source and incoherent sensor has been implemented. The block diagram of the system is shown in Figure 1.

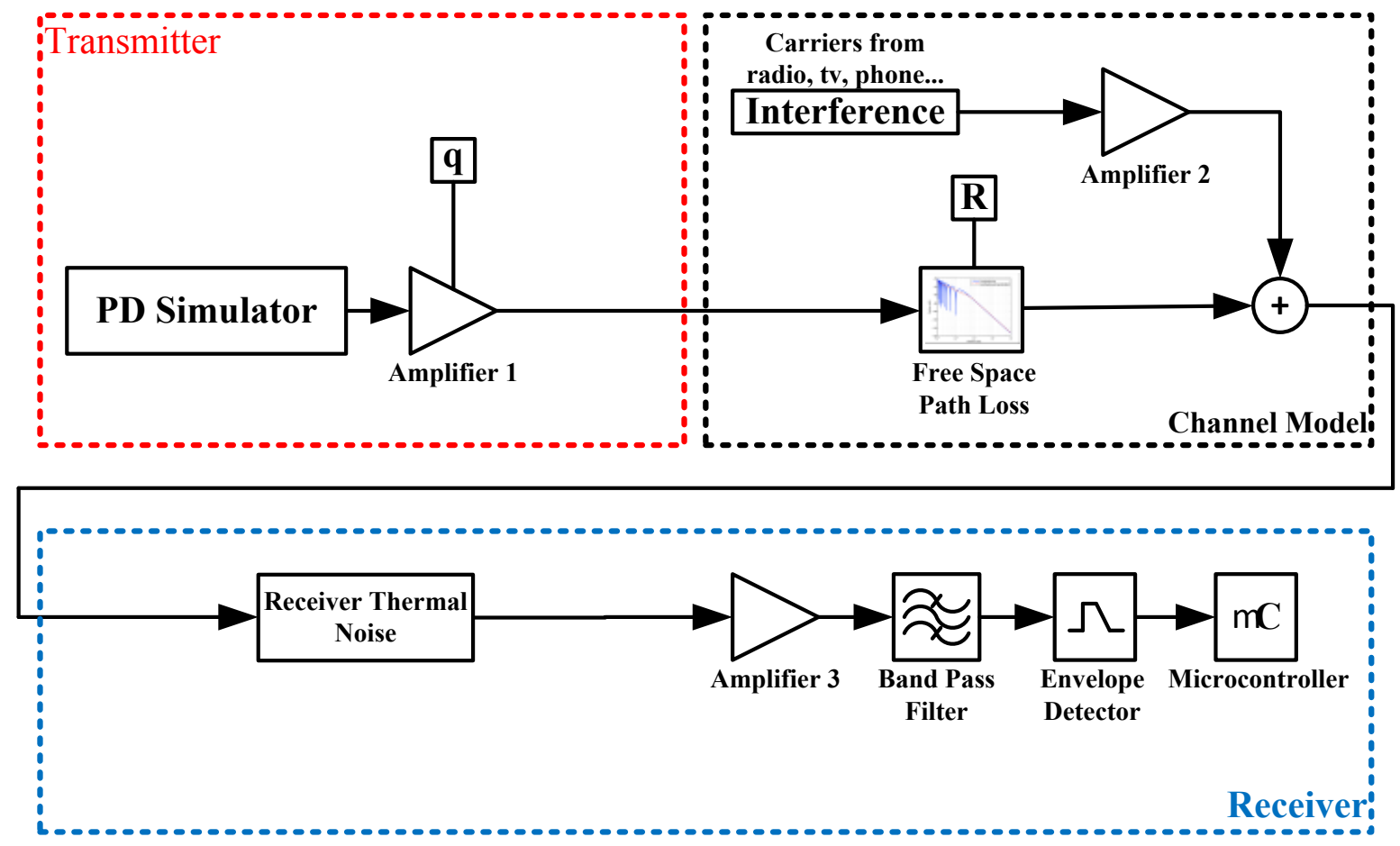

Fig. 1. Block diagram of the simulated system, where $\mathrm{q}$ is the pulse charge and $\mathrm{R}$ is the distance between source and sensor.

\section{MODEL}

The simulated PD is generated by replaying the radiated $\mathrm{RF}$ field measured using a disk-cone antenna connected to a 5Gs/s Tektronix oscilloscope model TDS 5104B. The simultaneous PD current pulses were also recorded on a separate channel of the oscilloscope using a high-frequency current transformer (HFCT). The PD current pulses were also recorded using a commercial (Doble Lemke LDS-6) PD measurement system. The equipment used for the measurements is shown in Figure 2. The diskcone is a monopole variation of the biconical antenna. It is low-gain, omnidirectional, linearly-polarised and has wide bandwidth [6]. The partial discharge generator was made from an acrylic cylinder and plane, parallel, disc electrodes. Two nylon screws are used to set the distance between the electrodes which are connected to the high-voltage power supply. Details of the PD 
generator are given in $[4,7]$. A measured PD pulse is shown in Figure 3.

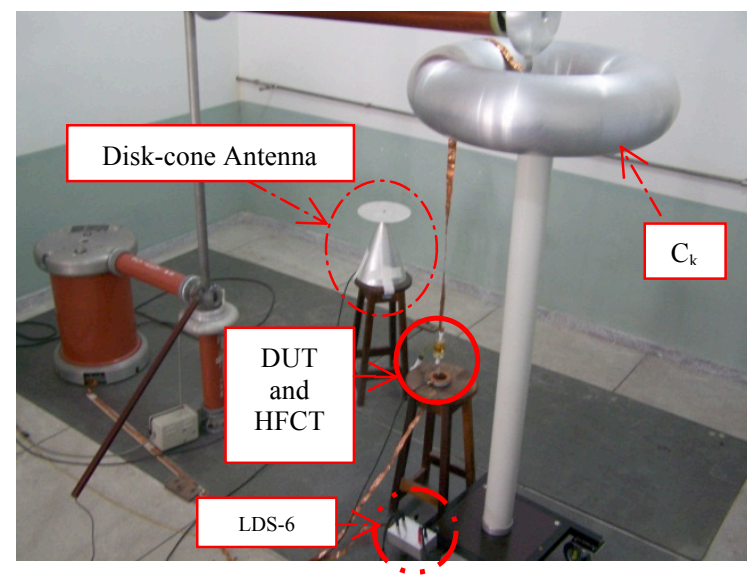

Fig.2. Measurement equipment.

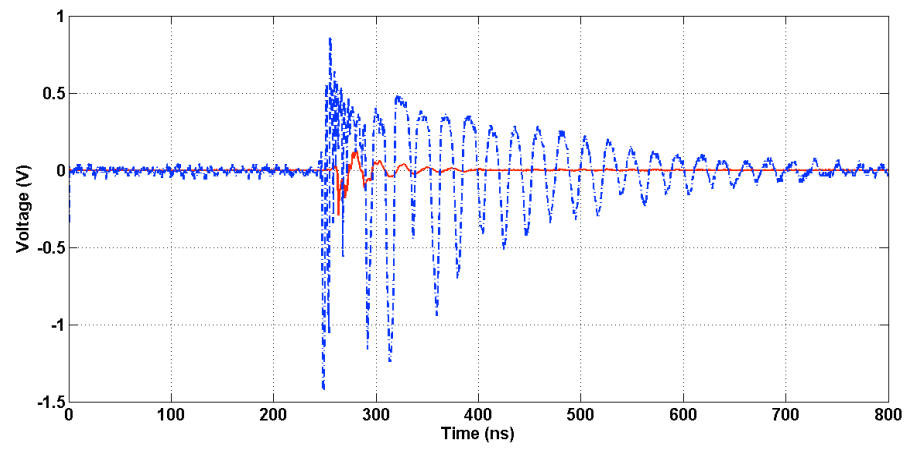

Fig.3. Pulses obtained using antenna (dashed line) and HFCT (solid line).

The SNR of the PD measurement was $24.6 \mathrm{~dB}$. The noise inherent in the replayed PD is therefore assumed to be negligible (since the SNR of the PD signal in the WSN receivers is likely to be much less than this).

The first amplifier 1 in the chain of subsystems in Figure 1 is used to set the PD signal power. This power is determined from the PD intensity (in $\mathrm{pC}$ ) using a relationship to be determined empirically by measuring the $\mathrm{PD}$ signal power received using a disk-cone antenna and spectrum analyzer at a range of $1 \mathrm{~m}$ from a $\mathrm{PD}$ source whose intensity in $\mathrm{pC}$ is being measured using a LDS-6 instrumentation [8]).

The frequency response of the radiating structure is, in general, unknown and will depend on the particular geometry and materials at the site of the PD and the arrangement of conductors and insulators leading away from it. Since a replay model of the PD signal is being used here no explicit model of the radiating structure frequency response is needed. This is because the effect of the radiating structure frequency response has already been accounted for in the measurement. A similar argument applies to the receive antenna. In a simulation where the PD is generated from a physical model instead of an empirical measurement then the frequency responses of both radiating structure and receive antenna would need to be explicitly represented in the simulation.

The path loss model used for this simulation is simply that expected for free-space propagation [9].
Narrowband, coherent, interference is represented by a set a (unmodulated) sinusoids. The number, frequency and power of these interferers will vary from location to location and from time to time. A sample from this random process was obtained by measurement in the HV laboratory at the Federal University of Campina Grande (UFCG), Figure 4. The measurements were made over a band $10-2000 \mathrm{MHz}$, using a disk-cone antenna and a spectrum analyzer. Table 1 shows the carrier frequencies and powers extracted from the measurements and used in the simulation reported here.

\begin{tabular}{|c|c|}
\hline Frequency $(\mathrm{MHz})$ & Power $(\mathrm{dBm})$ \\
\hline 29.9 & -39.69 \\
\hline 69.7 & -53.70 \\
\hline 94.58 & -45.27 \\
\hline 158.3 & -52.52 \\
\hline 189.1 & -56.52 \\
\hline 209 & -57.63 \\
\hline 228.9 & -58.53 \\
\hline 253.8 & -58.27 \\
\hline 293.6 & -59.64 \\
\hline 477.7 & -59.67 \\
\hline 527.4 & -60.2 \\
\hline 880.6 & -56.81 \\
\hline 1750 & -17.00 \\
\hline
\end{tabular}

Table 1. Frequency and power from carriers used at the simulation.

The amplifier 2 following the coherent interference generator allows the gross power of the interference to be changed. The frequencies and powers of the individual carriers will be specific to a particular location and (probably less so) specific to a particular time but, provided that there are (at least) several independent carriers which are not dominated by a small subset (one or two), then it is probably gross interference power which is important in assessing the plausibility of the detection system being considered. This gross power will also be location and time dependent and so a model of the spatial and temporal statistics of this power within a particular geographical region would be useful in assessing the proportion of sensors that would operate effectively for a given proportion of time. (This is equivalent to determining the proportion of PD events of a particular intensity that would be successfully detected.) One of the important mechanisms by which a single carrier might come to dominate for small fractions of time is the presence of a mobile telephone handset close to a sensor. This scenario is not unlikely in the context of operations and maintenance engineers, for example, visiting the site where a sensor network is deployed. An interference measurement was therefore made with a mobile telephone placed $1 \mathrm{~m}$ from the measurement antenna. (This is the dashed, blue, curve in Figure 4.) 


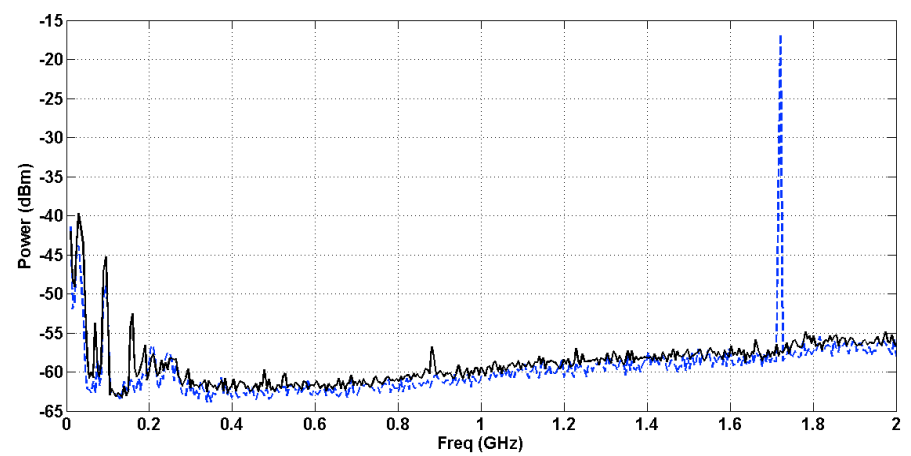

Fig. 4. Measured interference environment: black solid curve measured in absence of mobile phone handset, blue dashed curve measured in presence of mobile phone handset

Since significant changes in PD intensity relate to trends measured over (at least) many hours, and more probably days or weeks, such 'rare' events would almost certainly be detected and flagged as not significant by the data processing algorithms. Figure 5 shows the structure of the interference block.

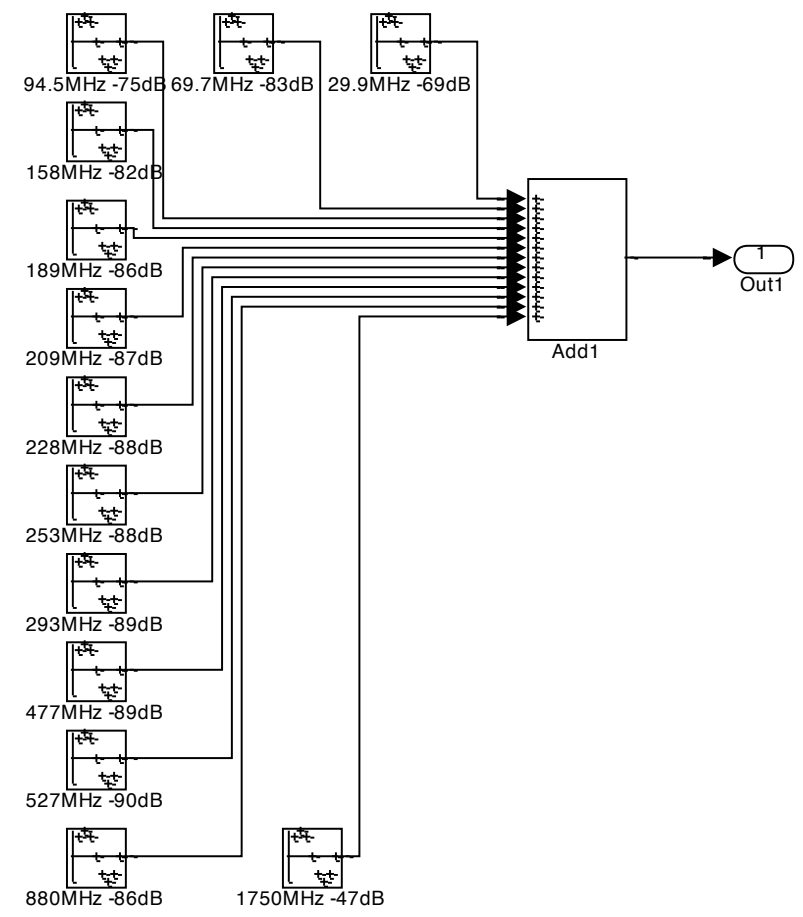

Fig. 5. Sum of sinusoids representing carriers from wireless communications and similar devices.

Additive white Gaussian noise (AWGN) is added at the receiver input assuming an antenna temperature of $300 \mathrm{~K}$.

Amplifier 3 is included, principally, to allow internal receiver noise to be added. The noise Figure of this amplifier for the results presented here was chosen to be $3.0 \mathrm{~dB}$. The gain was chosen to be $0 \mathrm{~dB}$ since the simulated envelope detector is ideal. In a practical implementation of the receiver the amplifier will need to have sufficient gain for the resulting signal to drive a practical envelope detector. The bandwidth of the receiver is limited by a sixth-order band-pass filter with lower a cut-off frequency of $50 \mathrm{MHz}$ and an upper cut-off frequency of $600 \mathrm{MHz}$. The envelope detector is an ideal amplitude demodulator comprising squaring, low-pass filtering and square-rooting operations, Figure 6. The low-pass filter in the envelope detector is of fourth-order with a cutoff frequency of $50 \mathrm{MHz}$.

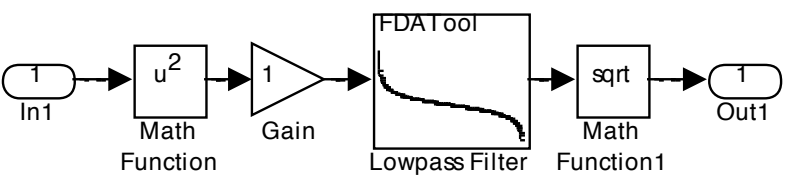

Fig. 6 Envelope detector

\section{RESULTS}

Figure 7 shows the pre-detection peak SNR as a function of sensor range from the PD source.

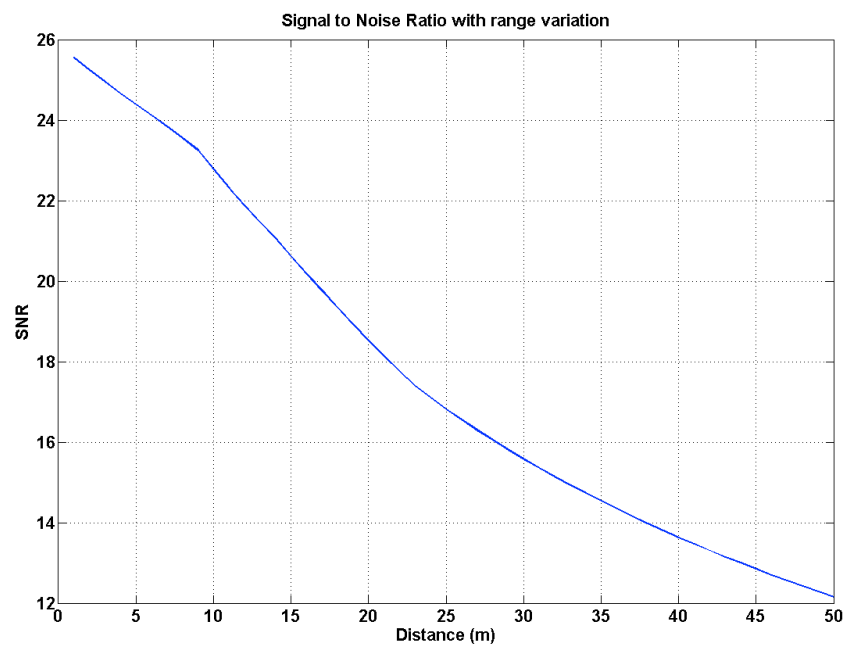

Fig. 7. SNR for a range variation of $50 \mathrm{~m}$.

Figure 7 suggests that the peak SNR is adequate up to distances of at least $50 \mathrm{~m}$. Figures $8 \mathrm{a}, \mathrm{b}, \mathrm{c}$ and $\mathrm{d}$ show the measured pulse at a distance of 1, 10, 25 and $50 \mathrm{~m}$ from the PD source.

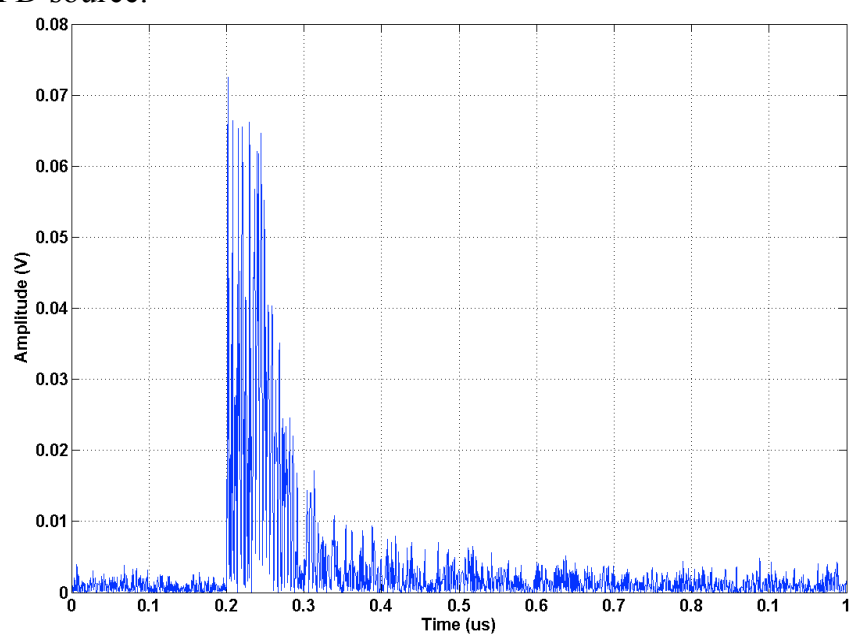

(a) 


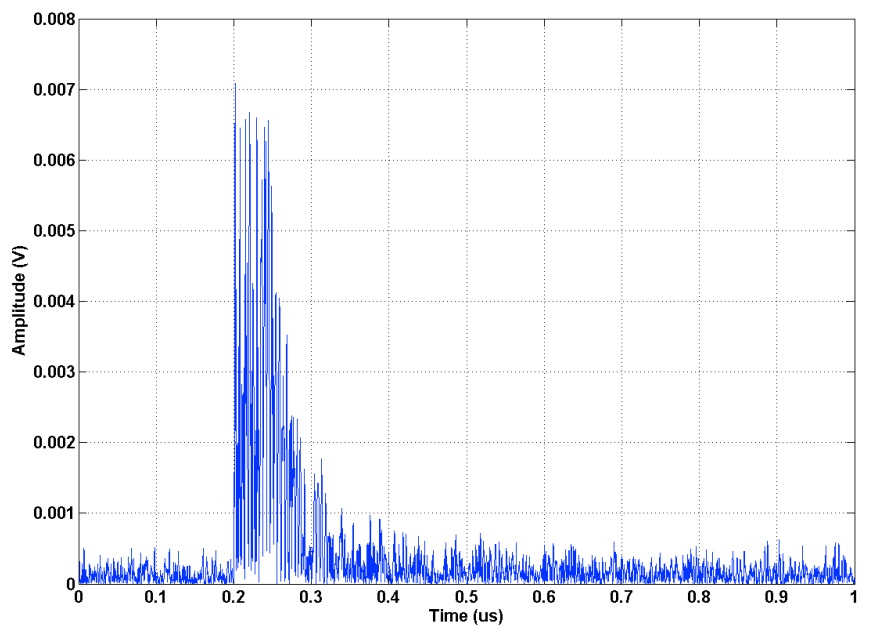

(b)

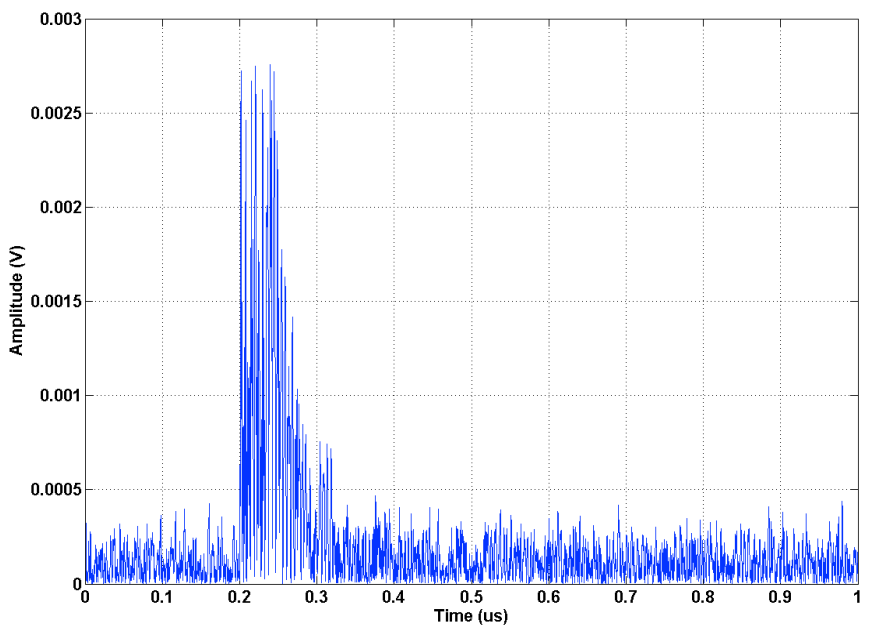

(c)

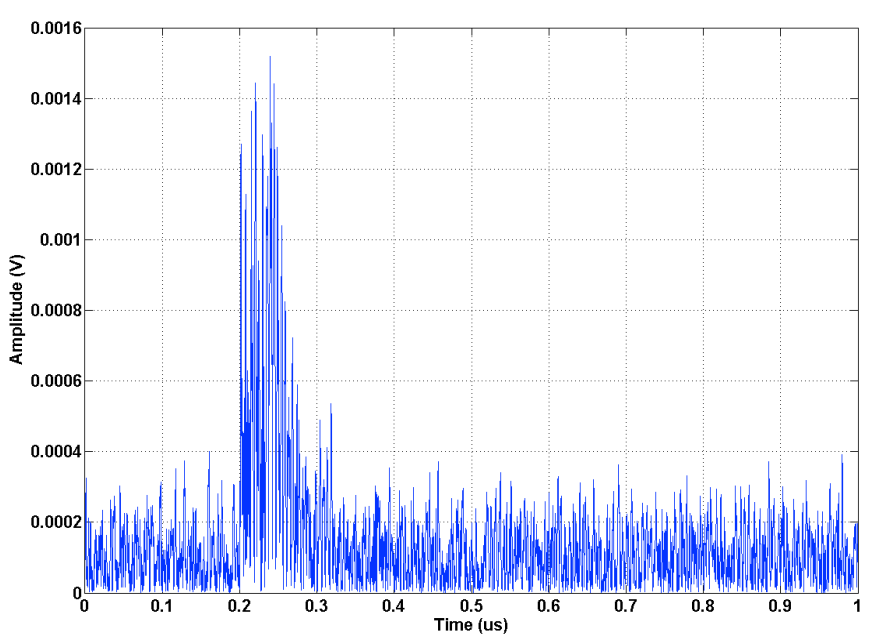

(d)

Fig. 8. Measured signal at a distance of (a) $1 \mathrm{~m}$, (b) $10 \mathrm{~m}$, (c) $25 \mathrm{~m}$ and (d) $50 \mathrm{~m}$.

Figures 9(a), (b), (c) and (d) show the expected envelope detector output superimposed on the pre-detected signal.

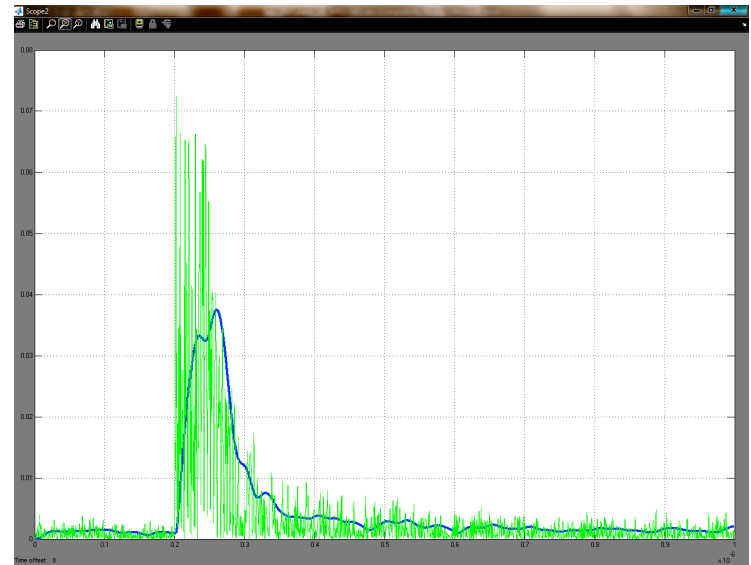

(a)

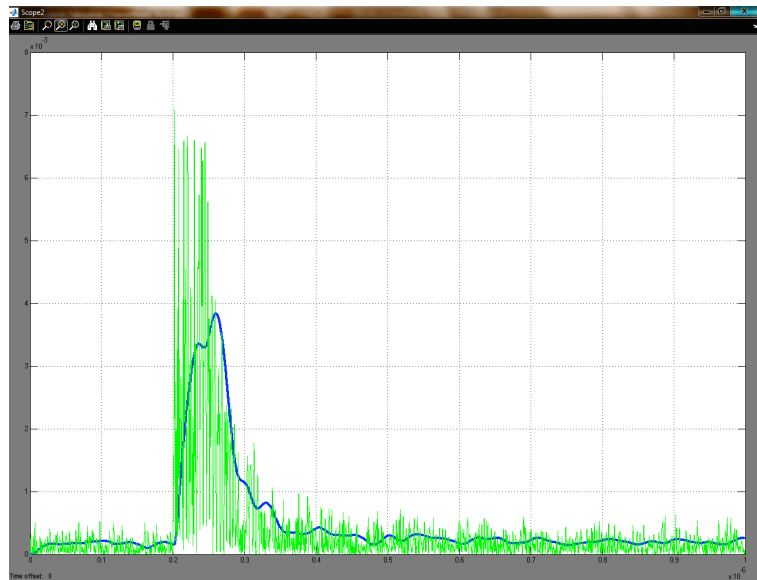

(b)

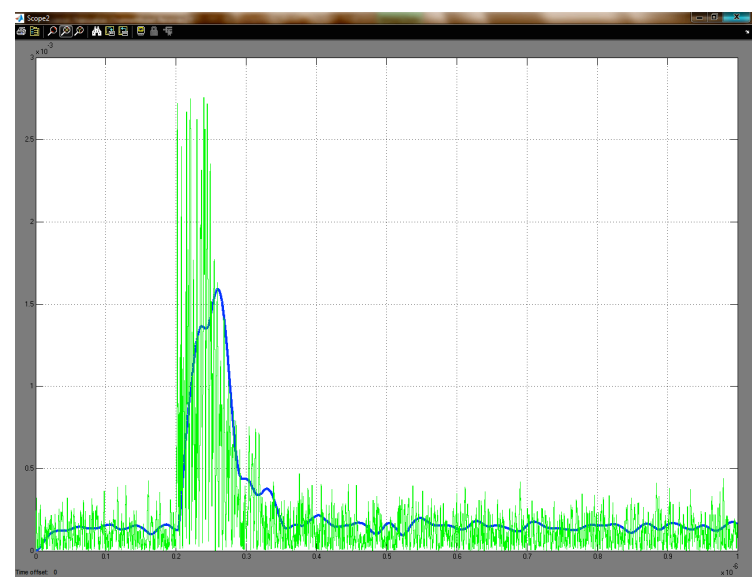

(c) 


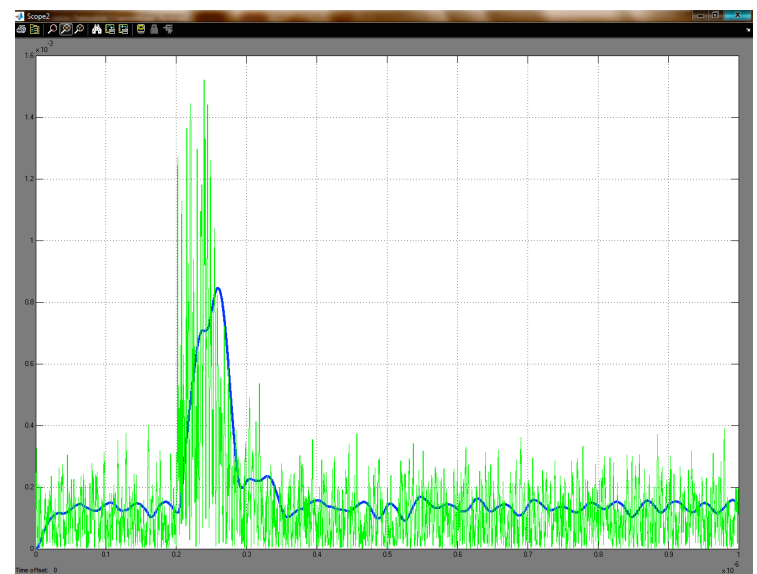

(d)

Fig. 9. Pre-detection PD signal (green dashed curve) and post-detection signal (blue solid line) for a sensor at a range of (a) $1 \mathrm{~m}$, (b) $10 \mathrm{~m}$, (c) $25 \mathrm{~m}$ and (d) $50 \mathrm{~m}$.

\section{CONCLUSIONS}

A simulation has been used to investigate the plausibility and sensitivity of a free-space radiometric receiver employing envelope detection. Measured data was used to model a PD source. A free-space path loss model has been used to simulate transmission loss and narrowband coherent interference has been modeled by extracting a set of carriers from measured background spectra. The pre-detection bandwidth of the receiver is $550 \mathrm{MHz}$. The post-detection (video) bandwidth is $50 \mathrm{MHz}$. This appears to yield satisfactory SNR up to a range of at least $10 \mathrm{~m}$. The envelope detector allows sampling frequency to be reduced at the expense of SNR and thus provides a convenient mechanism for trading these quantities. As low a sampling rate as possible is desirable (consistent with the minimum required SNR) since this reduces the cost of the ADC. It also reduces the power required to (i) drive the ADC and (ii) process data locally and/or transmit the data to a collection node. This is important if energy harvesting is to be used to provide this power.

The feasibility of this receiver depends on the envelope detector since the previous stages of the system were already tested with real components. Its sensitivity allows measurements within a range up to $12 \mathrm{~m}$, from PD sources of $600 \mathrm{pC}$, with $-30.45 \mathrm{~dB}$ at $12 \mathrm{~m}$. This is just an example, but for measurements in a real environment a more sensitive receiver should be used, by the use of an amplifier before the envelope detector. Nevertheless, these results are sufficiently encouraging to warrant further development of the envelope detector approach to radiometric PD detection.

\section{ACKNOWLEDGMENT}

One of the authors thanks CNPq in Brazil for a scholarship within the programme Science without Boarders and the authors thank EPSRC in the UK for support via grant (EP/J015873/1). The authors also thank the Universities of Strathclyde and Huddersfield for their support in executing this research.

\section{REFERENCES}

[1] Moore P J, Portugues I and Glover I A: Radiometric location of partial discharge sources on energised high-voltage plant, IEEE Trans. in Power Delivery, vol. 20, no. 3, pp. 2264-2272, July 2005.

[2] de Souza Neto, J. M. R., Cavalcanti, T. C. M., da Rocha Neto, J. S. and Glover, Ian (2012) Partial discharge detection and location in electricity substations for the self-diagnosing smart grid. In: XIX Congresso Brasileiro de Automatica (CBA 2012), 2-6th September 2012, Campina Grande, Brazil.

[3] de Souza Neto, J. M. R., Macedo, E C T, Rocha Neto, J S Da, Da Costa, E G, Bhatti, S A, Glover, I A, 2012, 'Partial discharge location using unsynchronized radiometer network for condition monitoring in $\mathrm{HV}$ substations - a proposed approach' Journal of Physics Conference Series, vol 364, no. 1.

[4] Macedo, E.C.T., de Souza Neto, J M R, Villanueva, Juan, Guedes, E. C., Freire, Raimundo and Glover, Ian (2012) Partial Discharge Estimation Based on Radiometric and Classical Measurements. In: IEEE International Instrumentation and Measurement Technology Conference, 13-16th May 2012, Graz, Austria.

[5] T Pinpart and M D Judd, "Differentiating between partial discharge sources using envelope comparison of UHF signals", IET Science, Measurement and Technology, Vol. 4, Issue 5, pp. 256-267, September 2010 .

[6] de Souza Neto, J.M.R.; de Macedo, E.C.T.; Cavalcanti, T.C.M.; Guedes, E.C.; da Rocha Neto, J.S.; Glover, I.A., "Development of a partial discharge emulator for calibration of a radiometric PD detection system,"Instrumentation and Measurement Technology Conference (I2MTC), 2012 IEEE International, vol., no., pp.2398,2403, 13-16 May 2012.

[7] de Souza Neto, J.M.R.; de Macedo, E.C.T.; Batista, M.L.N.; Cavalcanti, T.C.M.; Guedes, E.C.; da Rocha Neto, J.S.; Glover, I.A., "Early progress in the development of a radiometric PD location system,"Instrumentation and Measurement Technology Conference (I2MTC), 2012 IEEE International, vol., no., pp.129,133, 13-16 May 2012, Graz, Austria.

[8] Double Lemke. Prospect LDS-6, Jun 2013. http://www.doblelemke.eu/fpdf/product.pdf.php?product_id=3

[9] Proakis, J., Digital Communications. New York: McGraw-Hill, 2001. 